\title{
AN ACCURATE SOLUTION OF THE POISSON EQUATION BY THE LEGENDRE TAU METHOD
}

\author{
MUHAMMAD I. SYAM
}

Department of Mathematics

Yarmouk University

Irbid, JORDAN

(Received November 1, 1995 and in revised form March 24, 1996)

\begin{abstract}
A new Tau method is presented for the two dimensional Poisson equation Comparison of the results for the test problem $u(x, y)=\sin (4 \pi x) \sin (4 \pi y)$ with those computed by Haidvogel and Zang, using the matrix diagonalization method, and Dang-Vu and Delcarte, using the Chebyshev collocation method, indicates that our method would be more accurate
\end{abstract}

KEY WORDS AND PHRASES: Poisson equation, Legendre polynomials, Tau method 1991 AMS SUBJECT CLASSIFICATION CODES: 65

\section{INTRODUCTION}

Haidvogel and Zang [1] developed a matrix diagonalization method for the solution of the twodimensional Poisson equation. This method is efficient but requires a preprocessing calculation of the eigenvalues and eigenvectors which limits the accuracy of the solution to that of the preprocessing calculations, especially at large $N$ values

Dang-Vu and Delcarte [2] developed a Chebyshev collocation method for solving the same problem Their method has the same accuracy as the matrix diagonalization method when $N$ is small and it is more accurate when $N$ is large In this paper we present a new alternative method for solving

$$
\begin{gathered}
\Delta u(x, y)=u_{x x}+u_{y y}=f(x, y), x, y \in(-1,1) \\
u( \pm 1, y)=u(x, \pm 1)=0 .
\end{gathered}
$$

which is more accurate than the above two methods

\section{PRELIMINARIES}

In this section we give a basic definition and some facts which we use hereafter

DEFINITION 1. The Legendre polynomial $\left\{L_{k}(x), k=0,1, \ldots\right\}$ are the eigenfunctions of the singular Sturm-Liouville problem

$$
\left(\left(1-x^{2}\right) L_{k}^{\prime}(x)\right)^{\prime}+k(k+1) L_{k}(x)=0, \quad x \in[-1,1] .
$$

Like other orthogonal polynomials the Legendre polynomials satisfy many relationships perhaps the most basic one is the orthogonality relation

$$
\int_{-1}^{1} L_{n}(x) L_{m}(x) d x=(n+0.5)^{-1} \delta_{n m}
$$

for $n \geq 1$ and 


$$
\delta_{n m}=\left\{\begin{array}{ll}
1 & \text { if } n=m \\
0 & \text { if } n \neq m
\end{array} .\right.
$$

Other properties of Legendre polynomials include the recursion relation

$$
L_{n+1}(x)=\frac{2 n+1}{n+1} x L_{n}(x)-\frac{n}{n+1} L_{n-1}(x)
$$

for $n \geq 1$ and the endpoint relation

$$
L_{n}( \pm 1)=( \pm 1)^{n}
$$

Suppose that $f(x) \in C^{2}[-1,1]$ and $f^{\prime \prime \prime}(x)$ is a piecewise continuous function on $[-1,1]$ Then for

$$
\mathscr{L} f(x)=\frac{d^{2}}{d x^{2}} f(x)
$$

we have that

$$
\mathscr{L} f(x)=\sum_{n=0}^{\infty} f_{n}^{(2)} L_{n}(x)
$$

.converges uniformly on $[-1,1]$ where

$$
f_{n}^{(2)}=\left(n+\frac{1}{2}\right) \sum_{\substack{p=n+2 \\ p \neq n \text { even }}}[p(p+1)-n(n+1)] f_{p},
$$

and

$$
f_{n}=(n+0.5) \int_{-1}^{1} f(x) L_{n}(x) d x .
$$

The coefficients also satisfy the recursion relation

$$
\frac{f_{n-1}^{(q)}}{2 n-1}-\frac{f_{n+1}^{(q)}}{2 n+3}=f_{n}^{(q-1)}, \quad n \geq 1, \quad q=1,2
$$

For more details, see Schwarz [3]

\section{LEGENDRE-TAU METHOD FOR SOLVING \\ TWO-DIMENSIONAL POISSON EQUATION}

The basic topics of this section involve the Legendre-Tau method to discretize a class of linear boundary value problems of the form of Problem (ll 1 ). To explain the total procedure both analytic and numerical results are presented

Referring to the boundary value problem (1.1) approximate $u$ and $f$ in terms of Legendre polynomials as

$$
u_{N}(x, y)=\sum_{k=0}^{N} a_{k}(x) L_{k}(y)
$$

and

$$
f_{N}(x, y)=\sum_{k=0}^{N} b_{k}(x) L_{k}(y)
$$

For the approximate solution $u_{N}$, the residual is given by

$$
R_{N}\left(u_{N}\right)=\Delta u_{N}(x, y)-f_{N}(x, y) .
$$


Thus, the residual can be written as

$$
R_{N}\left(u_{N}\right)=\sum_{k=0}^{N}\left[a_{k}^{(2)}(x)+a_{k}^{\prime \prime}(x)-b_{k}(x)\right] L_{k}(y),
$$

where $a_{k}^{(2)}$ is given in (2.4) and $a_{k}^{\prime \prime}(x)$ is the second derivative of $a_{k}(x)$ with respect to $x$ As in a typical Galerkin scheme we generate $(N-1)$ second order ordinary differential equations by orthogonalizing the residual with respect to the basis functions $L_{k}(y)$

$$
\left(R_{N}, L_{k}(y)\right)=\int_{-1}^{1} R_{N} L_{k}(y) d y=0, \quad \text { for } \quad k=0: N-2 .
$$

This leads to the elementwise equation

$$
a_{k}^{(2)}(x)+a_{k}^{\prime \prime}(x)=b_{k}(x) .
$$

Since

$$
a_{k}(x)=r_{k} a_{k-2}^{(2)}(x)+s_{k} a_{k}^{(2)}(x)+w_{k} a_{k+2}^{(2)}(x), \quad \text { for } \quad k=2: N
$$

so

$$
\begin{gathered}
a_{k}=r_{k}\left(b_{k-2}-a_{k-2}^{\prime \prime}\right)+s_{k}\left(b_{k}-a_{k}^{\prime \prime}\right)+w_{k}\left(b_{k+2}-a_{k+2}^{\prime \prime}\right), \quad \text { for } \quad k=2: N, \\
r_{k}=\frac{1}{(2 k-3)(2 k+1)}, \quad w_{k}=\frac{1}{(2 k+5)(2 k+3)}, \quad s_{k}=\frac{-4 k-4}{(2 k+1)^{2}(2 k+3)}, \\
r_{k}=0 \quad \text { if } k>N+2, \quad s_{k}=0 \quad \text { if } \quad k>N \quad \text { and } \quad w_{k}=0 \quad \text { if } \quad k>N-2 .
\end{gathered}
$$

For simplicity, let us assume that $N$ is even positive integer. Let $D^{2}=\frac{d^{2}}{d x^{2}}$ be the differential operator Since

$$
u_{N}(x, \pm 1)=0=\sum_{k=0}^{N}( \pm 1)^{k} a_{k}(x)
$$

so

$$
a_{0}(x)+a_{2}(x)+\ldots+a_{N}(x)=0
$$

and

$$
a_{1}(x)+a_{3}(x)+\ldots+a_{N-1}(x)=0 .
$$

Thus, we have the following two systems

$$
\left(A_{e}+D^{2} B_{e}\right) \underline{a_{e}}=\underline{R_{e}}
$$

and

$$
\left(A_{0}+D^{2} B_{0}\right) \underline{a_{0}}=\underline{R_{0}}
$$

where $\underline{a_{e}}=\left(a_{0}, a_{2}, \ldots, a_{N}\right)^{T}, \underline{a_{0}}=\left(a_{1}, a_{3}, \ldots, a_{N-1}\right)^{T}$,

$$
A_{e}=\left[\begin{array}{ccccc}
1 & 1 & \ldots & 1 & 1 \\
0 & 1 & \ldots & 0 & 0 \\
& & \ldots & & \\
0 & 0 & \ldots & 1 & 0 \\
0 & 0 & \ldots & 0 & 1
\end{array}\right], \quad B_{e}=\left[\begin{array}{ccccccccc}
0 & 0 & 0 & 0 & \ldots & 0 & 0 & 0 & 0 \\
r_{2} & s_{2} & w_{2} & 0 & \ldots & 0 & 0 & 0 & 0 \\
0 & r_{4} & s_{4} & w_{4} & \ldots & 0 & 0 & 0 & 0 \\
& & & & \ldots & & & & \\
0 & 0 & 0 & 0 & \ldots & r_{N-4} & s_{N-4} & w_{N-4} & 0 \\
0 & 0 & 0 & 0 & \ldots & 0 & r_{N-2} & s_{N-2} & 0 \\
0 & 0 & 0 & 0 & \ldots & 0 & 0 & r_{N} & 0
\end{array}\right],
$$




$$
\begin{gathered}
A_{0}=\left[\begin{array}{lllll}
1 & 1 & \ldots & 1 & 1 \\
0 & 1 & \ldots & 0 & 0 \\
0 & 0 & \ldots & & \\
0 & 0 & \ldots & 0 & 1
\end{array}\right], B_{o}=\left[\begin{array}{ccccccccc}
0 & 0 & 0 & 0 & \ldots & 0 & 0 & 0 & 0 \\
r_{3} & s_{3} & w_{3} & 0 & \ldots & 0 & 0 & 0 & 0 \\
0 & r_{5} & s_{5} & w_{5} & \ldots & 0 & 0 & 0 & 0 \\
& & & \ldots & & & & \\
0 & 0 & 0 & 0 & \ldots & r_{N-5} & s_{N-5} & w_{N-5} & 0 \\
0 & 0 & 0 & 0 & \ldots & 0 & r_{N-5} & s_{N-3} & 0 \\
0 & 0 & 0 & 0 & \ldots & 0 & 0 & r_{N-1} & 0
\end{array}\right] \\
\underline{r_{e}}
\end{gathered}
$$

Since $\left\{L_{k}(y), k=0,1, \ldots, N\right\}$ is linearly independent over $\Re$ and $u_{N}( \pm 1, y)=0$, we see that

$$
\underline{a_{e}}( \pm 1)=\underline{0} \text { and } \underline{a_{o}}( \pm 1)=\underline{0} \text {. }
$$

From equations ( 34$)-(3.6)$, we see that the two systems are similar. For this reason, we will discuss the solution of the following system

$$
\left(A_{e}+D^{2} B_{e}\right) \underline{a_{e}}=\underline{R_{e}}, \quad \underline{a_{e}}( \pm 1)=\underline{0} .
$$

Multiply both sides of the differential equation (3.7) by

to get

$$
A_{e}^{-1}=\left[\begin{array}{cccccc}
1 & -1 & -1 & \ldots & -1 & -1 \\
0 & 1 & 0 & \cdots & 0 & 0 \\
0 & 0 & 1 & \cdots & 0 & 0 \\
0 & 0 & 0 & \cdots & 1 & 0 \\
0 & 0 & 0 & \cdots & 0 & 1
\end{array}\right]
$$

$$
\left(I+D^{2} C_{e}\right) \underline{a_{e}}=\underline{q_{e}}, \quad \underline{a_{e}}( \pm 1)=0 .
$$

Let $X$ and $R$ be two matrices of sizes $(N / 2+1) \times(N / 2+1)$ such that $X_{2 j}$ and $R_{2 j}$ are the coefficients of $x^{j-1}$ in the $i^{\text {th }}$ component of $\underline{a}_{e}$ and $\underline{q_{e}}$ respectively. Let

$$
q_{+}=\left[\begin{array}{llll}
1 & 1 & \ldots & 1
\end{array}\right] \text { and } q_{-}=\left[\begin{array}{lllllll}
1 & -1 & 1 & -1 & \ldots & (-1)^{n / 2}
\end{array}\right]
$$

be $1 \times(N / 2+1)$ matrices Then system (3 8) can be written as

$$
\left[\begin{array}{c}
I+D^{2} C_{e} \\
q^{+} \\
q^{-}
\end{array}\right] X=\left[\begin{array}{l}
R \\
\underline{0} \\
\underline{0}
\end{array}\right]
$$

Multiply both sides of the differential equation (3.9) by

$$
\left[\begin{array}{lll}
I+D^{2} C_{e}^{T} & q_{+}^{T} & q_{-}^{T}
\end{array}\right]
$$


to get

$$
\left(I+D^{2}\left(C_{e}+C_{e}^{T}\right)+D^{4} C_{e}^{T} C_{e}+q_{+}^{T} q_{+}+q_{-}^{T} q_{-}\right) X=\left(I+D^{2} C_{e}^{T}\right) R
$$

THEOREM 3.1. The matrix $G=I+q_{+}^{T} q_{+}+q_{-}^{T} q_{-}$is a nonsingular matrix

PROOF. Let $\lambda$ be any eigenvalue of the matrix $G$ associated with the eigenvector $x$ such that $x^{T} x=1$

$$
\begin{gathered}
\lambda=\lambda x^{T} x=\lambda x^{T} G x \\
=x^{T} x+\left(x q_{+} x\right)^{T}\left(x q_{+} x\right)+\left(x q_{-} x\right)^{T}\left(x q_{-} x\right) \geq 1 .
\end{gathered}
$$

Then, the smallest eigenvalue of $G$ is at least 1 , which implies that $G$ is nonsingular matrix Now, multiply both sides of the differential equation $(310)$ by $Q=\left(I+q_{+}^{T} q_{+}+q_{-}^{T} q_{-}\right)^{-1}$ to get

$$
\left(I+D^{2} Q\left(C_{e}^{T} C_{e}\right)+D^{4} Q C_{e}^{T} C_{e}\right) X=Q\left(I+D^{2} C_{e}^{T}\right) R
$$

It is easy to see that

$$
\begin{aligned}
& Q=\left(I-\alpha q_{+}^{T} q_{+}\right)\left(I-\beta q_{-}^{T} q_{-}\left(I-\alpha q_{+}^{T} q_{+}\right)\right), \\
& \alpha=\frac{1}{1+q_{+} q_{+}^{T}}, \quad \beta=\frac{1}{1+q_{-}\left(I-\alpha q_{+}^{T} q_{+}\right) q_{-}^{T}} .
\end{aligned}
$$

For more details, see Hager [4]

Since each component of $Q\left(I+D^{2} C_{e}^{T}\right) R$ is a polynomial of degree at most $N / 2$, so we will approximate the solution of equation (3.11) by

$$
X=\sum_{i=0}^{\llbracket N / 4 \rrbracket}(-1)^{2} S^{2}\left(Q+D^{2} C_{e}^{T}\right) R
$$

where $S=D^{2} Q\left(C_{e}^{T}+C_{e}\right)+D^{4} Q C_{e}^{T} C_{e}$ and $\llbracket N / 4 \rrbracket$ is the largest integer less than or equal $N / 4$ Let $H$ be the transition matrix from the basis $\beta_{1}=\left\{L_{0}(x), L_{1}(x), \ldots, L_{[N / 4]+1}\right\}$ to the basis $\beta_{2}=\left\{1, x, \ldots, x^{[N / 4]+1}\right\}$ for the space

$$
P_{\llbracket N / 4 \rrbracket+1}=\{f: f \text { is a polynomial of degree } \leq \llbracket N / 4 \rrbracket+1\}
$$

with usual addition and scaler multiplication Let $\Gamma$ be the matrix of the differential operator $D^{2}: P_{[N / 4]+1} \rightarrow P_{[N / 4]-1}$ using the standard basis. Thus, the algorithm for computing $X$ is given as follows

\section{ALGORITHM 3.1.}

$\begin{array}{ll}\text { INPUT: } & \text { The matrices } R, C_{e}, Q, H \text { and } \Gamma \\ \text { OUTPUT: } & \text { The matrix } X . \\ \text { STEP 1 } & \text { Compute } R_{1}=\Gamma R^{T} ; R_{2}=Q\left(R+C_{e}^{T} R_{1}^{T}\right) \\ \text { STEP 2 } & X=R_{2} . \\ \text { STEP 3 } & \text { For } i=1: \llbracket N / 2 \rrbracket+1 \text {, do steps 4-6. } \\ \text { STEP 4 } & R_{3}=\Gamma R_{2}^{T} ; R_{4}=\Gamma^{2} R_{2}^{T} \\ \text { STEP 5 } & R_{2}=Q\left(\left(C_{e}^{T}+C_{e}\right) R_{3}^{T}+C_{e}^{T} C_{e} R_{4}^{T}\right) . \\ \text { STEP 6 } & X=X+(-1)^{2} R_{2} . \\ \text { STEP 7 } & \text { Stop. }\end{array}$

\section{NUMERICAL RESULT}

In this section, we give two experimental examples to show how Algorithm (3.1) works nicely Also, comparison of the results for the test problem $u(x, y)=\sin (4 \pi x) \sin (4 \pi y)$ with those computed by Haidvogel and Zang, using the matrix diagonalization method, and Dang-Vu and Delcarte, using the Chebyshev collocation method will be done 
All the calculations are realized using the 486 IBM computer Programs are written in double precision

EXAMPLE 4.1. Consider the following boundary value problem for $-1<x<1$ and $-1<y<1$

$$
\begin{aligned}
u_{x x}(x, y)+u_{y y}(x, y) & =-32 \pi^{2} \sin (4 \pi x) \sin (4 \pi y) \\
u( \pm 1, y) & =0=u(x, \pm 1)
\end{aligned}
$$

The exact solution is $u(x, y)=\sin (4 \pi x) \sin (4 \pi y)$ We will study the relation between the number of terms in the approximation solution $N$ and the error in the approximation $\epsilon_{N}$ This relation is given in Table (1)

Table (1)

\begin{tabular}{|c|c|}
\hline \multicolumn{2}{|c|}{ Maximum Absolute Error $\epsilon$ as a function of $N$} \\
\hline \hline$N$ & $\epsilon$ \\
\hline 16 & $3.23 \times 10^{-2}$ \\
\hline 24 & $6.87 \times 10^{-6}$ \\
\hline 32 & $4.31 \times 10^{-11}$ \\
\hline 40 & $1.0 \times 10^{-16}$ \\
\hline
\end{tabular}

EXAMPLE 4.2. Consider the following boundary value problem for $0<x<1$ and $0<y<1$

$$
\begin{gathered}
u_{x x}(x, y)+u_{y y}(x, y)=f(x, y) \\
u(1, y)=u(0, y)=0=u(x, 0)=u(x, 1)
\end{gathered}
$$

where $f(x, y)=32\left[\left(x^{2}-x\right)\left(x+y^{2}-1\right)+\left(y^{2}-y\right)\left(y+x^{2}-1\right)\right] e^{2 x+2 y-2}$

The exact solution is $u(x, y)=16\left(x^{2}-x\right)\left(y^{2}-y\right) e^{2 x+2 y-2}$ We will study the relation between the number of terms in the approximation solution $N$ and the error in the approximation $\epsilon_{N}$ This relation is given in Table (2) In this case, first we will use the following transformation to the square $[0,1] \times[0,1]$ into the square $[-1,1] \times[-1,1]$

$$
z=2 x-1, \quad w=2 y-1
$$

Table (2)

\begin{tabular}{|c|c|}
\hline \multicolumn{2}{|c|}{ Maximum Absolute Error $\epsilon$ as a function of $N$} \\
\hline$N$ & $\epsilon$ \\
\hline 10 & $1.01 \times 10^{-9}$ \\
\hline 12 & $5.16 \times 10^{-12}$ \\
\hline 16 & $1.04 \times 10^{-15}$ \\
\hline
\end{tabular}

From Table (1) and Table (2), we see that our method is an accurate method Compared with the Haidvogel-Zang method and Dang-Delcarte method our method should generate more accurate results at large $N$ values

\section{REFERENCES}

[1] HAIDVOGEL, D B. and ZANG, T., The accurate solution of Poisson's equation by expansion in Chebyshev polynomials, J. Comput. Phys. 30, 167 (1979).

[2] DANG-VU, H. and DELCARTE, C., An accurate solution of the Poisson equation by Chebyshev collocation method, J. Comput. Phys. 104 (1993), 211-220.

[3] SCHARZE, H.R., Numerical Analysis, John Wiley and Sons, New York, 1989

[4] HAGER, W W , Updating the inverse of a matrix, Siam Review, 31, 2 (1989), 221-239 


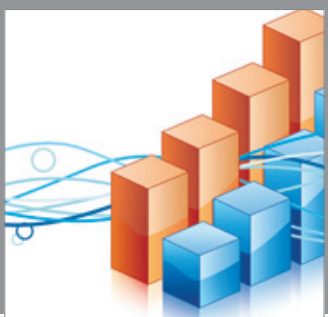

Advances in

Operations Research

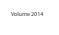

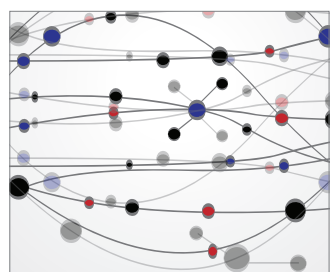

\section{The Scientific} World Journal
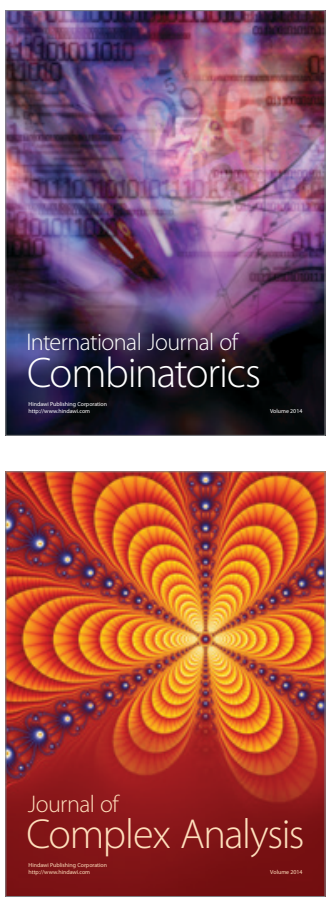

International Journal of

Mathematics and

Mathematical

Sciences
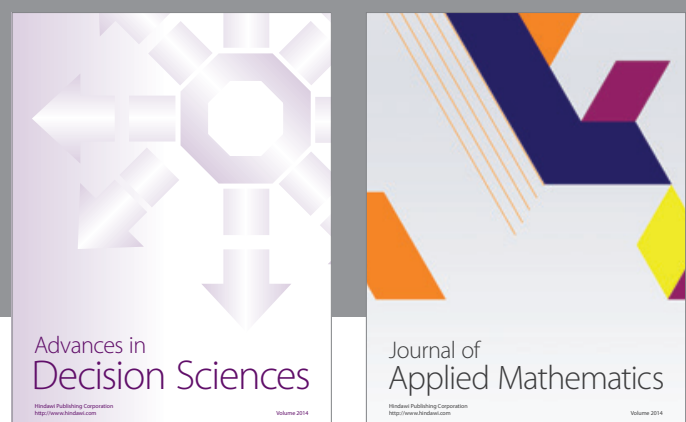

Journal of

Applied Mathematics
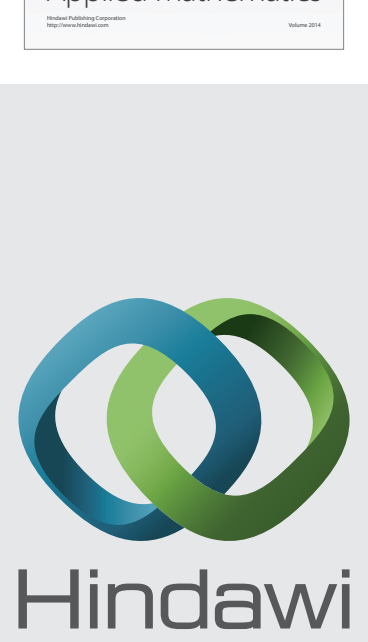

Submit your manuscripts at http://www.hindawi.com
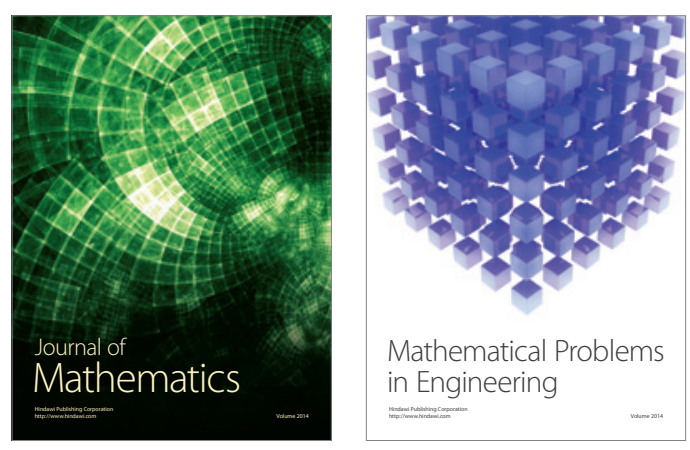

Mathematical Problems in Engineering
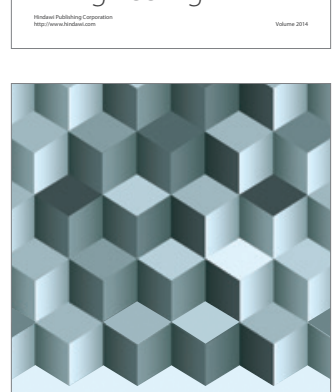

Journal of

Function Spaces
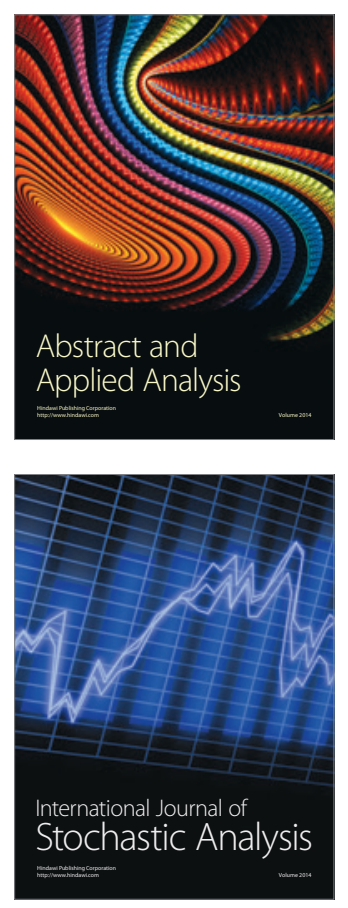

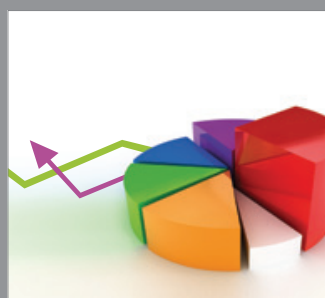

ournal of

Probability and Statistics

Promensencen
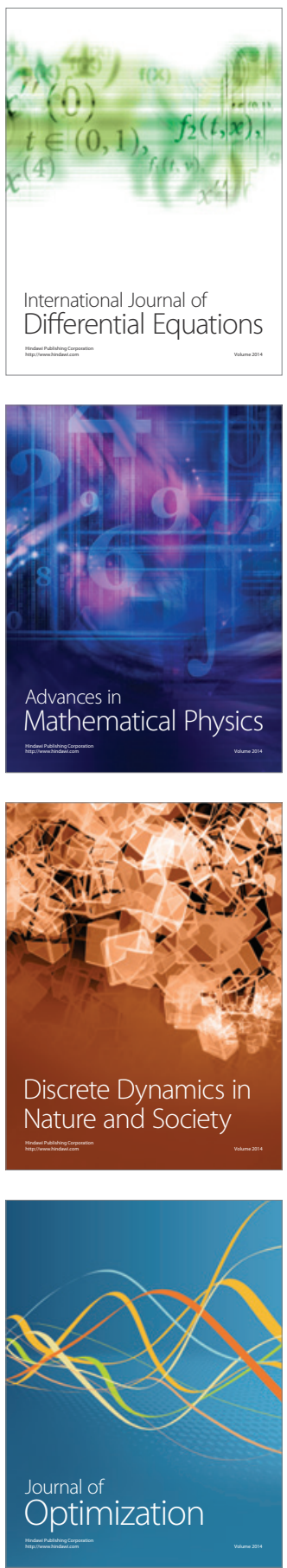